\title{
Household appliance usage recommendation based on demand forecasting and multi-objective optimization
}

\author{
Recomendação de utilização de aparelhos domésticos com base em predição de demanda e \\ otimização multi-objetivo
}

Recomendación de uso de electrodomésticos basada en la predicción de la demanda y la optimización multiobjetivo

\author{
Allan Rivalles Souza Feitosa \\ ORCID: https://orcid.org/0000-0002-7850-5223 \\ Universidade Federal de Pernambuco, Brazil \\ E-mail: arsf@cin.ufpe.br \\ Henrique Figueiroa Lacerda \\ ORCID: https://orcid.org/0000-0002-1296-9682 \\ Universidade Federal de Pernambuco, Brazil \\ E-mail: hfl@cin.ufpe.br \\ Wellington Pinheiro dos Santos \\ ORCID: https://orcid.org/0000-0003-2558-6602 \\ Universidade Federal de Pernambuco, Brazil \\ E-mail: wellington.santos@ufpe.br \\ Abel Guilhermino da Silva Filho \\ ORCID: https://orcid.org/0000-0002-7876-2756 \\ Universidade Federal de Pernambuco, Brazil \\ E-mail: agsfs@cin.ufpe.br
}

\begin{abstract}
Accelerated population growth in the 21st century and increased demand for energy sources, associated with climate change, have resulted in two main challenges: the search for sustainable energy sources and the need to find more efficient ways to use extant sustainable sources. The forecasting module provides an estimate of the future usage of these appliances and it is the source of the recommended module's suggestion. Time Series Forecasting techniques, such as Autoregressive Integrated Moving Average, Long-Short Term Memory (LSTM), Gated Recurrent Units, Echo State Networks (ESN), and Support Vector Regression, were tested for the predictive module. Multi-objective optimization techniques such as Non-Sorted Genetic Algorithm II (NSGA II), Multi-Objective Particle Swarm Optimization (MOPSO), Speed constrained Multi--objective Particle Swarm Optimization (SMOPSO), and Strength Pareto Evolutionary Algorithm two (SPEA2), for example, were tested for the Recommendation Module. The Forecasting and Recommendation module experiments were performed independently. In the Forecasting Module, the results and statistical tests revealed LSTM as the best- suited technique for forecasting the loads of the majority of the appliances tested (in this case seven) in terms of root mean square error. In the experiments performed for the recommendation module, NSGA II showed a higher overall performance compared to other metrics in terms of hyper volume of the Pareto Front generated. This work presents the potential of using both Predictive Models and Multi-Objective Optimization Techniques combined to reduce energy usage in household environments.
\end{abstract}

Keywords: Time series forecasting; Multi-objective optimization; Smart home systems; Recommendation systems.

\section{Resumo}

$\mathrm{O}$ crescimento populacional acelerado no século 21 e o aumento da demanda por fontes de energia, associados às mudanças climáticas, resultaram em dois desafios principais: a busca por fontes de energia sustentáveis e a necessidade de encontrar formas mais eficientes de usar as fontes sustentáveis existentes. O módulo de previsão fornece uma estimativa do uso futuro desses aparelhos e é a fonte da sugestão do módulo recomendado. Técnicas de previsão de série temporal, como Long--short Term Memory (LSTM), Gated Recurrent Units, Echo State Networks (ESN), e Support Vector Regression, foram testadas para o módulo preditivo. Técnicas de otimização multiobjetivo, como Non-Sorted Genetic Algorithm II (NSGA II), Multi-Objective Particle Swarm Optimization (MOPSO), Speed constrained Multi--objective Particle Swarm Optimization (SMOPSO), e Strength Pareto Evolutionary Algorithm two (SPEA2), por exemplo, foram testados para o Módulo de Recomendação. Os experimentos dos módulos de Previsão e Recomendação foram realizados de forma independente. No Módulo de previsão, os resultados e os testes estatísticos revelaram o LSTM como a técnica mais adequada para prever as cargas da maioria dos aparelhos testados (neste caso, sete) em termos de erro quadrático médio. Nos experimentos realizados para o módulo de recomendação, o NSGA II 
apresentou um desempenho geral superior em relação às outras métricas em termos de hipervolume da Frente de Pareto gerada. Este trabalho apresenta o potencial do uso de Modelos Preditivos e Técnicas de Otimização MultiObjetivo combinadas para reduzir o uso de energia em ambientes domésticos.

Palavras-chave: Previsão de séries temporais; Otimização multi-objetivo; Sistemas de casa inteligente; Sistemas de recomendação.

\section{Resumen}

El crecimiento demográfico acelerado en el siglo XXI y la mayor demanda de fuentes de energía, asociada al cambio climático, derivaron en dos desafíos principales: la búsqueda de fuentes de energía sostenibles y la necesidad de encontrar formas más eficientes de utilizar las fuentes sostenibles existentes. El módulo de pronóstico proporciona una estimación del uso futuro de estos dispositivos y es la fuente de la sugerencia del módulo recomendado. Se probaron técnicas de predicción de series de tiempo como Long--short Term Memory (LSTM), Gated Recurrent Units, Echo State Networks (ESN), e Support Vector Regression para el módulo predictivo. Técnicas de optimización multiobjetivo como Non-Sorted Genetic Algorithm II (NSGA II), Multi-Objective Particle Swarm Optimization (MOPSO), Speed constrained Multi--objective Particle Swarm Optimization (SMOPSO), and Strength Pareto Evolutionary Algorithm two (SPEA2), por ejemplo, se han probado para el módulo de recomendaciones. Los experimentos de los módulos de Predicción y Recomendación se llevaron a cabo de forma independiente. En el Módulo de Predicción, los resultados y las pruebas estadísticas revelaron al LSTM como la técnica más adecuada para predecir las cargas de la mayoría de los dispositivos probados (en este caso, siete) en términos de error cuadrático medio. En los experimentos llevados a cabo para el módulo de recomendación, el NSGA II presentó un desempeño general superior en relación al resto de métricas en términos de hipervolumen del Frente Pareto generado. Este trabajo presenta el potencial de utilizar modelos predictivos y técnicas de optimización multiobjetivo combinadas para reducir el uso de energía en entornos domésticos. Palabras clave: Predicción de series de tiempo; Optimización multiobjetivo; Sistemas domésticos inteligentes; Sistemas de recomendación.

\section{Introduction}

The accelerated population growth of the 21 st century and the resulting demand for more energy sources associated with climate change have brought two main challenges: first, the need to develop greener electricity sources that do not harm the environment and, at the same time, meet the main demands, and second, the need to manage efficiently energy consumption by avoiding electricity wastage and stimulating more conscious energy usage. In 2018, electricity generation was responsible for $38 \%$ of the global energy -related CO2 emissions. This represented a growth of $4 \%$ relative to 2017 . This rate is mainly due to the economic growth of the population and consequent increase on demand. Moreover, the increasing demand is also a result of climate change such as warmer summers and the consequent increased reliance on air conditioning, as well as colder winters and the need for increased heating in specific regions of the world (International Energy Agency, 2019).

A Home Energy Management System (HEMS) is capable of managing connected house appliances in order to decrease electricity usage during peak hours and avoid electricity wastage (Shareef et al., 2018).

Several techniques have been implemented for smart control HEMS. Heuristic and evolutionary techniques such as Bacterial Foraging Optimization, Dynamic Programming, and Genetic Algorithms, have been used to schedule appliance usage for out-of--peak periods and reduce the waiting time for said use (M. U. Khalid \& Javaid, 2018) (Yuce et al., 2016) (Liu et al., 2016). Additionally, machine learning -based techniques such as Artificial Neural Networks (ANN) and Recurrent Neural Networks (RNN), as well as deep learning -based techniques have been implemented to forecast appliance usage and then perform a variety of actions such as automating the operation of appliances, predicting which appliances will be used during peak hours (Din et al., 2018), or recommending new patterns in appliance usage.

The implementation of Smart HEMS has been presented by several works as a solution to reduce electricity usage and/or address user comfort by predicting their behavior patterns and automating tasks. However, few works are concerned with recommending actions to the user instead of forcing the appliances to be turned off or simply automating their usage. A recommendation of an action has the advantage of being less invasive to the users' comfort and can be automated only if accepted by users. In addition, the dilemma between users' comfort and reduction of energy consumption is not widely approached. As a 
result, there is still a need to develop reliable smart HEMS recommendation techniques that are more accurate and realistic provided the reality of common users as well as less invasive to their comfort (Isnen et al., 2020).

Surveying the literature, few works approach the level of invasiveness of appliance usage rescheduling as regards the comfort of the user. The works that are concerned with this aspect normally model it by reducing the wait time for appliance usage or generating scheduling that is better aligned with the instant usage pattern of the appliances. The present work proposes a way of generating such recommendations by way of automatic forecasting of how each individual appliance will be used in a subsequent 24-hour period and then, based on the forecasting, generating usage recommendations that are balanced between energy saving and consumer comfort. That way recommendations are better aligned with the daily behavior of the user and, therefore, more comfortable for her/him and more likely to be deemed acceptable. Based on the above, the proposal of this work is a Household Appliance Usage Recommendation System based on forecasting of appliance usage and recommendations of new patterns of usage by considering the invasiveness of the recommendations to the comfort level of the user.

This work is organized as follows: section II presents related works in the literature; section III Describes the proposal of this work; in section IV, the experiments are described; section V presents the results and discussion; finally, the conclusions are discussed in section VI.

\section{Related Works}

There are works in the literature that touch on the prediction of household appliance loads by implementing mostly deterministic and machine learning methods, as well as works that approach the optimal scheduling of appliance usage in order to reduce energy wastage and increase the comfort level of the user. Works that approach forecasting and management systems and those describing approaches involving both prediction and management will be described further.

\subsection{Appliance -usage Forecasting}

Load forecasting plays an important role in several aspects of HEMS and is a field of study that has received a lot of attention in recent years. The objective of these techniques is to predict the future behavior of a load based on data about its past behavior (Khan et al., 2016). According to the application and necessity, the prediction of loads in HEMS can be performed in two ways: the prediction of the entire household load, also called Aggregated Forecasting, and the prediction of individual appliance loads, also called Disaggregated Forecasting. A few examples where deterministic and machine learning techniques have been implemented to generate those predictions will be presented in the present literature review.

The forecasting of disaggregated individual appliance loads is usually a more difficult task than predicting the whole aggregated residential load (Kong et al., 2018). Kumar et al. (2018) show implementations of recurrent neural networks to aggregated loads forecasting. In their work, they implement Long Short-Term Memory (LSTM) and Gated Recurrent Unit (GRU) nets to forecast the electricity consumption of residences given the past aggregated data about them. However, aggregated load forecasting can also benefit from the presence of disaggregated load data. Kong et al. (2018) show the potential of LSTM for aggregated load forecasting by collecting past data from both individual appliances and aggregated loads.

In literature, both deterministic and machine learning- based techniques can be used to predict disaggregated loads and time series forecasting. The works described in Oprea et al. (2018) and Gajowniczek and Zabkowski (2014) show the implementation of the Non-linear Autoregressive Exogenous (NARX) model, Neural Networks (NN), and the Support Vector Machine (SVM), along with different techniques for NN weight adjustments and different kernels for SVM for appliance usage prediction, to forecast individual appliance loads. Besides those techniques, the recurrent neural networks also have been proven to be good options for forecasting individual appliance loads. The work described in Kong et al. (2019) shows the performance of LSTM for forecasting individual appliance loads. 
There are few works in the literature that deal with the potential of Echo State Networks (ESN) (Jaeger, 2001) for disaggregated load forecasting. Shi et al. (2016), for example, show the implementation of several ESN topologies for forecasting the energy consumption of a building. As a result, this work will also consider ESN as a forecasting technique in its experiments.

The appliance usage recommendations of this work are generated for each individual appliance. Therefore, the forecasting and recommendation steps will be implemented for each individual appliance load. The main techniques highlighted in the appliance -usage forecasting literature review will be tested in order to find the best performance in the conditions of the experiments performed in this work.

\subsection{Home Energy Management Systems}

The Home Energy Management Systems comprises the inclusion of sensors and actuators in buildings and the consequent capacity of controlling and monitoring the usage of these appliances, aiming at energy efficiency, users' comfort, health and wellness, and other applications. Among those applications, one that has been widely studied is energy efficiency (Leitao et al., 2020). When the objective is to reduce the electric bill, there are two main applications of HEMS: Consumption Reduction, which aims to take actions to decrease the usage of appliances, and Load Shifting, which focuses on reducing electric bills by rescheduling the usage of appliances to times when the cost of electricity is lower (Leitao et al., 2020). Several works have proposed stochastic, heuristic, and metaheuristic approaches with the objective of reducing energy costs mainly by scheduling appliance usage to out--of--peak hours, when energy is less costly.

In addition to considering renewable energy input and appliance scheduling, the works described in Dinh et al. (2020) and Dao et al. (2020) also include both grid energy usage and selling in the optimization process. They describe the implementation of a bio-inspired Particle Swarm Optimization and an Intensive Quadratic Programming, respectively, to optimize the scheduling of appliance usage. Despite taking into consideration additional constraints of interactions with grids and renewable sources, they have not considered the impact of the scheduling process on users' comfort.

Rescheduling appliance usage can lead to a level of discomfort for users due to discrepancies between when users' wish to use appliances and when they are available for usage. In the literature, there is still a gap in studies as regards the trade-off between user comfort and energy saving (Isnen et al., 2020).

In HEMS, the comfort of the users has been verified in different ways, such as waiting time, appliance usage, thermal limits, and usage profile similarity.

The work described in M. U. Khalid and Javaid (2018), A. Khalid et al. (2018), and Merdanoğlu et al. (2020) deals with the impact of appliance scheduling on users' comfort by reducing the waiting time for appliance usage. The user comfort level as waiting time was also implemented in Molla et al. (2019), which compares the performances of a meta-heuristic Grey Wolf Optimizer and Particle Swarm Optimization. Reducing the waiting time of postponed usage of appliances may not consider the behavior of the user in terms of the sequence in which he or she intends to use the appliance. Other than that, for some appliances, the waiting time may not be the best factor to address regarding user comfort.

The work described in Isnen et al. (2020) proposes a monthly limit of energy usage, predetermined or set by the user and a rule--based system to limit the usage of appliances to meet daily quotas of energy usage derived from a monthly objective. The authors consider the users' comfort by detecting the presence of the users and triggering a level of quota flexibility after reaching limits. Golmohamadi et al. (2019) and Merdanoğlu et al. (2020) show the scheduling of appliances considering also the users' thermal comfort as a range of temperatures predefined by the users. For the optimization tasks, they implemented a Forward-Backward Algorithm and a stochastic optimization to schedule the optimized usage of appliances.

In addition to optimal appliance scheduling and user comfort, the works proposed by Monyei et al. (2018), Shakeri et al. (2018), and Shafie-Khah and Siano (2018) also consider the usage of renewable sources of energy like photovoltaic and wind 
power generation, as well as energy storage devices. To model user comfort, they take into consideration several factors, such as room temperature levels and usage of storage, instead of shifting. However, none of these works take into consideration the similarity between the optimal schedules generated and the usual behavior of the users regarding appliance usage.

The work proposed by Veras et al. (2018) brings a multi-objective approach to address the trade-off between energy savings and user comfort. Two concurrent objective functions were simultaneously minimized: one modeled the cost of appliance usage by planning the schedule; the other modeled the comfort of the schedule by measuring the distance between the suggested schedule and the real appliance data usage over a period of time. This methodology assumed, in the second objective function, that the future usage of the appliances was going to be equal to the immediate past - which may not be valid in terms of forecasting appliance usage - leading to an inaccurate estimate of the future usage.

The literature promotes several ways of approaching the impact of rescheduling appliance usage at the level of user comfort. Some works claim that by decreasing the wait time for appliance usage, user comfort will be increased. However, recommending appliance usage in certain periods of the day, close to when the user normally uses her/his appliances, instead of only reducing the waiting time, may bring more familiar recommendations by considering the user's behavioral patterns. Also, the concern with thermal comfort exclusively may be invasive to the usage of other appliances, affecting the user's comfort directly.

The present work proposes a technique for recommending new ways of regulating household appliance usage. Rather than immediate past usage patterns, this technique considers: the usage of all appliances instead of focusing on thermal comfort; the alignment of the recommendations and the user's regular behavior; and generating recommendations based on forecasts of future usage.

\subsection{Prediction and Management Approaches}

The following works describe approaches for home appliance scheduling based on forecasting of appliance usage, similarly to the approach described in this work.

A feedforward deep neural network together with linear programming for optimizing the operation of appliances in a Smart Home Environment is described in Hossen et al. (2018). The forecasting was performed over past data by a feedforward deep neural network. The scheduling was modeled as an optimization problem that takes as constraints aspects such as minimal energy necessary for each appliance, maximum electricity usage, electricity production capacity, the preferences of the users regarding the usage patterns of the appliances (i.e. preferred appliances at each time of the day) and equipment flexibility - this last one being the constraint directly related to the users' comfort, whereby the user specifies which appliance should or should not be included in the scheduling. The comfort was addressed by the user's choice about which appliances to include in the optimization process and preferences of usage time of each appliance. Despite considering the invasiveness of the optimization of the appliances to the comfort level of the user, it could be considered invasive in terms of demanding information from the user regarding appliance usage preferences. An approach that is capable of considering the comfort of the user without demanding manual inputs about the user's preferences, could be considered less invasive in terms of both appliance usage and the user's manual interaction with the system.

A home appliance scheduling system based on ANN and Genetic Algorithm (GA) was proposed in Yuce et al. (2016). In their approach, a feedforward artificial neural network (FF-ANN) was trained to predict environmental factors (wind speed, solar radiation, air temperature, etc.) and user behavioral data as room occupancy and appliances dictate. Then, a Genetic Algorithm was implemented to generate optimized schedules based on the predictions generated by FF-ANN. The objective function evolved the forecasting generated by the FF-ANN and the cost of electricity resulting in the optimized schedule. The global optimal schedule generated by the GA was intended to be the one that would return the least grid electricity usage as 
predicted by FFANN. This approach can be very assertive in terms of prediction; however, the analyzed variables in the forecasting step may not always be available in common households. In addition to that, the approach is only concerned with reducing the electricity cost, which may affect the level of comfort of the user.

The proposed technique can also be more assertive in the recommendation due to the fact that it takes forecasting usage as in Hossen et al. (2018) and Yuce et al. (2016). Furthermore, it does not require manual interaction with the user and, in the recommendation process, takes into consideration the user's comfort.

\section{The Proposed Approach}

The proposal of present work consists in two main modules, described as follows:

- Prediction Module: The objective is to estimate how the load of an appliance will behave in the short--term. This estimate is performed using a Time-Series Forecasting technique, looking at past data about an appliance's load behavior in order to predict how the load will behave in the future - this is the basis for the next module.

- Recommendation Module: The output of the prediction module will be a time series which predicts how the appliance will be used in the next 24 hours. The objective of the recommendation module is, based on that prediction, to generate a recommendation. This is performed by a multi-objective optimization technique whereby the two concurrent objectives to be minimized are energy consumption of the recommendation and an approximated measure between the recommendation and the predicted load given by the forecasting module.

Taking into consideration that these recommendations are generated for each appliance individually, the abovementioned steps are performed for each appliance individually.

Figure 1: The Proposed Approach: Based on stored past usage data from the appliances, the prediction module estimates the future usage of appliances, and, based on this estimate, the recommendation module suggests new efficient ways of using said appliances.

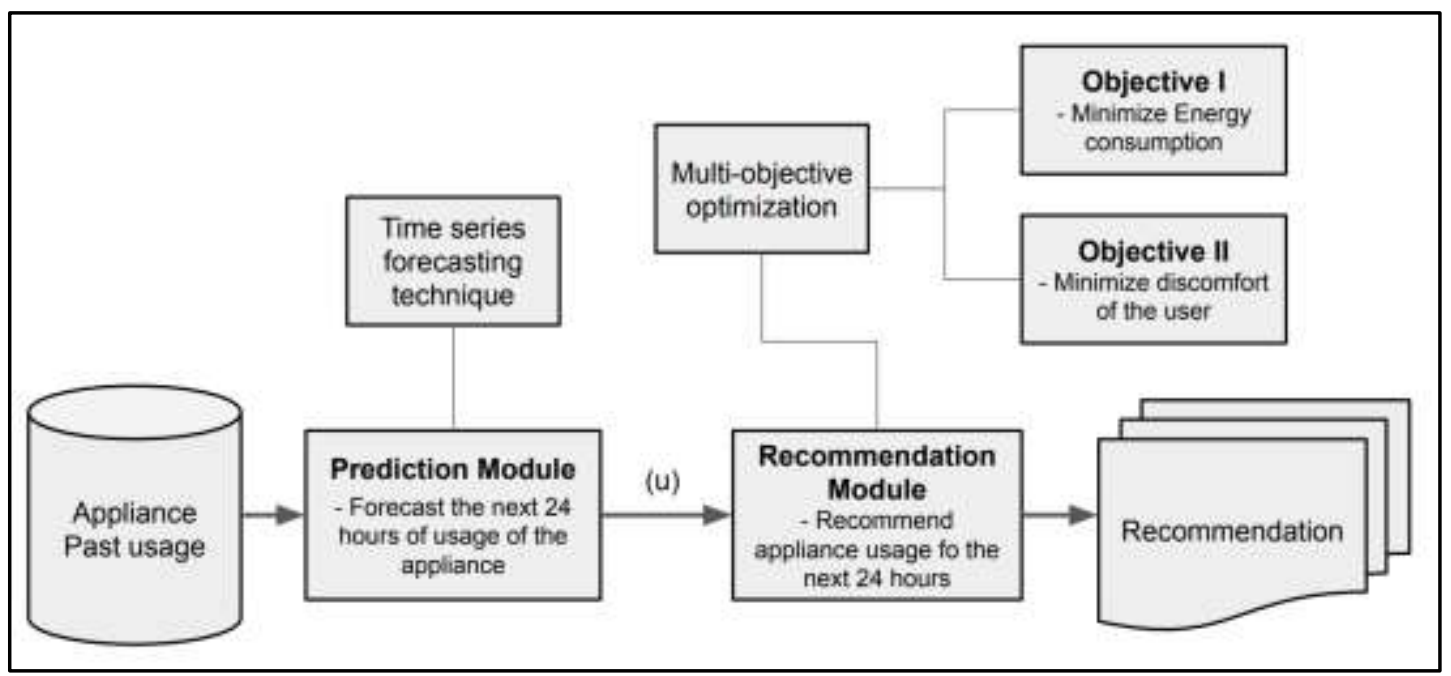

Source: Authors.

Each phase of the operation of the proposed technique, represented in Figure 1, will be explained as follows.

\subsection{Prediction Module}

A time -series forecasting technique, trained with past usage data of the appliance, gives a single- step -ahead forecast containing the predicted hourly aggregated power of the appliance for the next week. The result is a time series with a number 
of positions, each representing one hour. The predicted series for one week is represented by the following Equation 1:

$$
P=\{p 1, p 2, p 3, \cdots, p H\},
$$

where $\mathrm{H}$ is the size of the forecasting horizon, $\mathrm{P}$ is the forecasted time series, and pi is the aggregated power consumption of the appliance at the i--th hour, given by the sum of the per- second energy consumption of the appliance at the i--th hour.

\subsection{Recommendation Module}

The recommendation module operates with the forecasted time series for the appliance $\mathrm{P}$ and is based on a multi-objective optimization algorithm. The forecasted usage $\mathrm{P}$ is compared with the solution candidate in the comfort objective function, described in Equation 4. The following subsections explain the details of the multi-objective model.

\subsubsection{Solution Candidate}

The solution candidate contains the hourly consumption of an appliance over one week. Therefore, similarly to the forecasted usage of the appliance, it is represented by a vector containing 24 real values, each one representing the sum of the power consumption of the appliance for one hour. The solution candidate is represented in Equation 2, as follows:

$$
\mathrm{C}=\{\mathrm{c} 1, \mathrm{c} 2, \mathrm{c} 3, \ldots, \mathrm{cH}\}
$$

where $\mathrm{C}$ is a solution candidate and ci is the sum of the per -second energy consumption of the appliance at the i--th hour.

\subsubsection{Objective Functions}

The two concurrent objective functions are represented as follows.

The first one calculates the energy consumption of a solution candidate, which is the sum of 24 hours of hourly power usage by the appliance. This objective is forced to be minimized, representing the reduction of power usage by the user if she/he follows the recommendation. Energy saving is a sensitive topic when it comes to the user being pleased or not with receiving the recommendation to use less power. However, for the purpose of this work, it is assumed that the user will follow the recommendation. The energy consumption of the solution candidate is measured by the following equation:

$$
f_{1}(C)=\sum_{i=1}^{H} c_{i},
$$

where the whole energy consumption of the solution $\mathrm{C}$ is measured by summing its hourly power, and is intended to be minimized.

The second objective regards the proximity of the recommendation with the regular usage profile of the appliance by the user. The comfort of the user can be modeled for several points of view. One example is thermal comfort. However, For the purpose of this work the comfort level is interpreted as how similar the recommendation is to the way the user normally handles her/his appliances. The comfort level of one recommendation is quantified by the following equation:

$$
f_{2}(C)=\sqrt{\sum_{i=1}^{24}\left(c_{i}-p_{i}\right)^{2}},
$$

which measures the Euclidean distance between $\mathrm{C}$ and the future usage $\mathrm{P}$ which is the output of the forecasting step, interpreted as the discomfort level of the solution candidate, and also intended to be minimized.

The two objective functions explained before are used in a multi-objective optimization algorithm to generate solution candidates that will be treated as recommendations of how to use the appliances in the next 24 hours.

Algorithm 1 shows the implementation of the generation of one recommendation. Once the user requests a usage 
recommendation regarding the usage of one appliance for the next 24 hours, the recommendation is built as in the algorithm. The experiments realized in this work evolved the test of 6 prediction techniques and 4 multi-objective optimization techniques. However, in order to show the recommendation process (i.e. multi-objective optimization) the implementation of MOPSO (Coello \& Lechuga, n.d.) is shown in Algorithm 1.

Algorithm 1: Proposed technique.

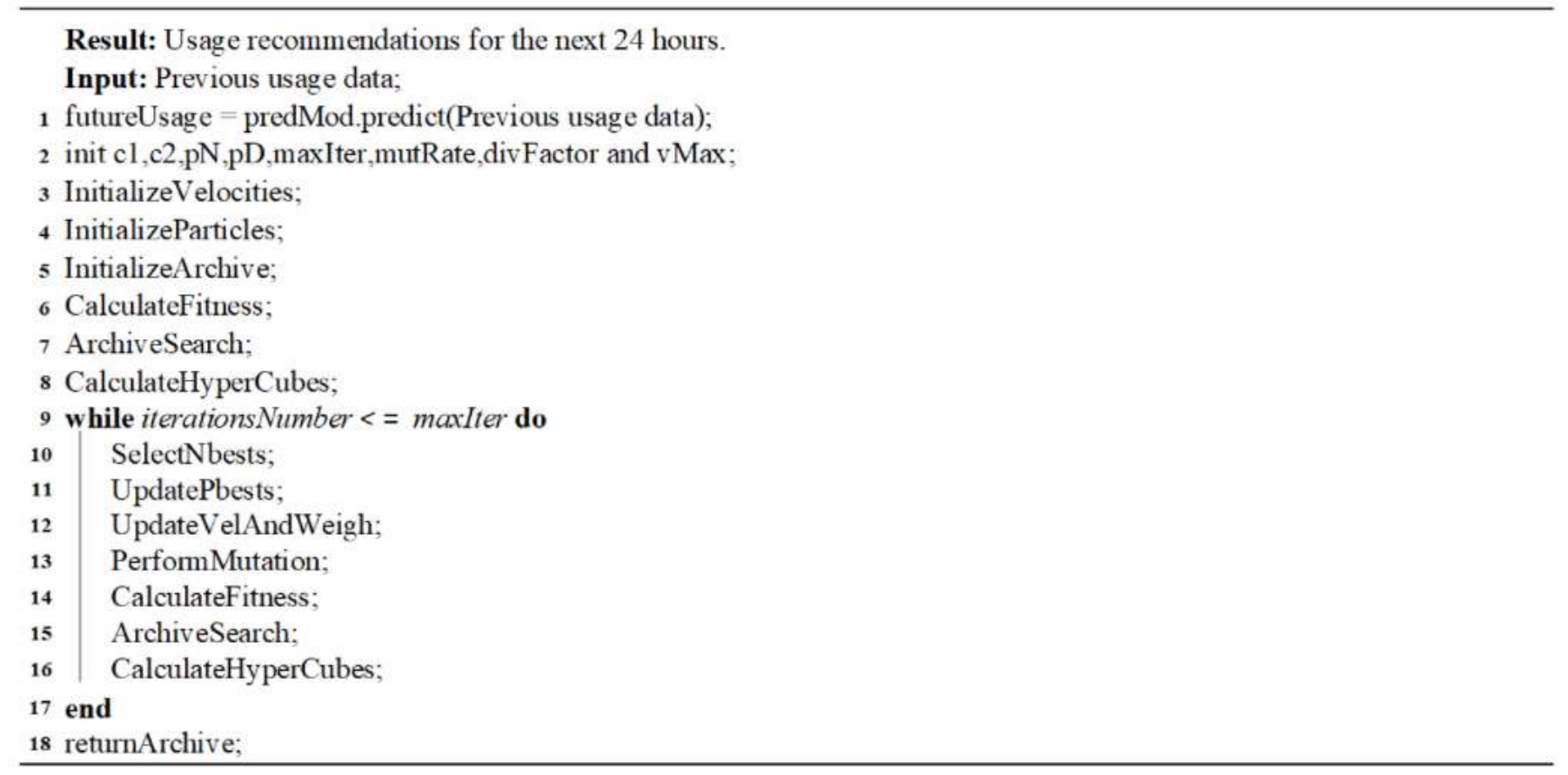

Source: Authors.

Each phase of algorithm 1 will be explained as follows:

1) Future usage: The future usage of the appliance is predicted by the forecasting algorithm. The algorithm works by receiving data from the last ' $\mathrm{J}$ ' hours of usage of the appliance and returning the prediction of the next 24 hours of usage of the appliance. 2) initialize $\mathrm{C} 1, \mathrm{C} 2, \mathrm{pN}, \mathrm{pD}$, maxIter, mutRate, divFactor and vMax: The parameters are initialized. Their description can be found in Table 1.

3) Initialize Velocities: The velocities matrix is initialized with random numbers. Its dimensions are $\mathrm{pN} x \mathrm{pD}$, as there is one velocity for each position of each particle.

4) Initialize Particles: Initialize $\mathrm{pN}$ particles with $\mathrm{pD}$ dimensions each. They are initialized with random continuous values.

5) Initialize Archive: The archive that stores the non-dominated solutions is initialized.

6) Calculate Fitness: Calculates the two objective function values for each particle.

7) Archive Search: The particles that meet at least one of the following requirements are included into the non-dominated solutions archive:

a) The archive is empty.

b) If the particle dominates one of the ones that are included in the archive it replaces the dominated one.

c) If the particle does not dominate and is not dominated by any other from the archive it is included into the archive.

8) Calculate Hypercubes: In the objectives space, split each objective into a number of subdivisions, resulting in hypercubes containing solutions fitness values.

9) While iterations Number $<=$ maxIterdo: Run the evolution of the swarm during a pre defined number of iterations. 
10) Select Nbests: For each particle select its Nbest, which is a random particle from a drawn hypercube. The hypercubes that have less solutions inside them have more probability of being selected. This is performed in the sense of enforcing diversity of solutions.

11) Select Pbests: The PBest for each particle is its best version considering the domination criteria. If the current version of the particle dominates the previous one, it will be its new PBest.

12) Update Vel and Weigh: The velocities and weights matrices are calculated for each particle. Those updating processes take in consideration the PBest and NBest defined in the previous two steps. The equation can be found in Coello \& Lechuga (n.d.).

13) Perform Mutation: Each particle has its weights changed by a random noise adding according to a probability defined in the mutRate parameter.

14) Calculate Fitness: Same as step 6.

15) Archive Search: Same as step 7.

16) Calculate Hyper Cubes: Same as step 8.

17) return Archive: Return the non-dominated solution resulting in the Archive. Those solutions will be treated as usage recommendations for the appliance being analyzed.

Table 1: MOPSO Parameters.

\begin{tabular}{|l|l|}
\hline Parameter & Description \\
\hline $\mathrm{c} 1$ and c2 & Local and social influence factors \\
\hline $\mathrm{pN}$ & Initial quantity of particles \\
\hline $\mathrm{pD}$ & Dimensions of each particle \\
\hline maxIter & Max number of iterations \\
\hline mutRate & Mutation rate \\
\hline divFactor & Hypercubes division factor \\
\hline vMax & Maximum velocity assumed by a particle \\
\hline
\end{tabular}

Source: Adapted from (Coello \& Lechuga, n.d.).

The final solutions in the Archive, in case of MOPSO, or the final multi-objective solutions in case of any other multi-objective optimization technique, will be interpreted as usage recommendations with different levels of comfort level and energy save.

\section{Experiments}

The experiments were performed by treating the forecasting and the recommendation modules separately. The forecast experiments aimed to find the best forecast technique for the conditions of this work, in terms of the smaller root mean square error between the forecasted series and a truth value. The recommendation experiment's objective was to find the best multi-objective technique in terms of hyper volume of the Pareto Front generated by the techniques.

The experiments were performed over a publicly available dataset described in Kleiminger et al. (2015). The prediction and recommendation experiments are performed on 7 appliances from the data set. The appliances are: Fridge, Dryer, Coffee Machine, Kettle, Washing Machine, Desktop Computer, and Freezer. 
The data was collected in a second by second based sampling rate for 4 months from September 15, 2012, to January 23, 2013.

\subsection{Forecasting}

In the forecasting step, the evaluation of the performances are performed by blocked cross validation (blocked cv) described by Cerqueira et al. (2019) and Bergmeir and Benítez (2012) for train and test split.

Figure 2: Blocked Cross Validation. A $\mathrm{k}$ fold without shuffling is used in the training set and once the best model configuration is found, it is then tested on the test set.

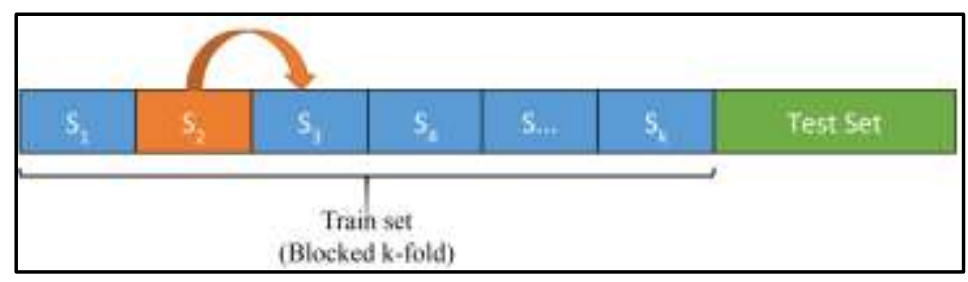

Source: Authors.

Figure 2 illustrates the train and test sets in blocked cv. First the whole set of samples is split in train and test groups. Then the whole training of the technique is performed in the train set by performing $\mathrm{k}$ fold cross validation without data shuffling in order to keep the temporal relationship between data. After finding the best hyperparameter configuration, the model is trained with the whole train data without cross validation and tested in the test set. In Figure 2, S is a subset of the training data split by $\mathrm{k}$-fold, and $\mathrm{K}$ is the number of folds in which the train set is split (usually 10). The fold $\mathrm{S} 2$ is the one being used to test the model in the k-th of $\mathrm{k}$ times. The final train error is given by the average of the obtained Root-Mean-Square-Error (RMSE) for each of the folds. RMSE is calculated as in Equation 5.

$$
\text { RMSE }-\sqrt{\frac{1}{n} \sum_{t=1}^{n}\left(y_{t}-\hat{y}_{t}\right)^{2}} .
$$

In Equation 5, $\mathrm{y}$ is test- ground truth, and $\mathrm{y}^{\wedge}$ is the forecasted load of the appliance.

The techniques tested in the forecasting step are Autoregressive Integrated Moving Average (ARIMA) described originally by Box et al. (1994), Support Vector Regression (SVR) for time series forecasting (Cao, 2003), Long-Short Term Memory (Hochreiter \& Schmidhuber, 1997), Gated Recurrent Units (Chung et al., 2014), and Echo State Networks (Jaeger, 2001).

The forecast is performed in a single step ahead, based on the last $\mathrm{J}$ hours, to predict the future $\mathrm{H}$ hours of usage of the appliance. A sliding window with fixed window and horizon forecasts is implemented to extract the samples from the training and testing data. Figure 3 shows an example of the sliding window.

Figure 3: Sliding window example. The prediction window () data is used as input, and the horizon $(H)$ is used as target output.

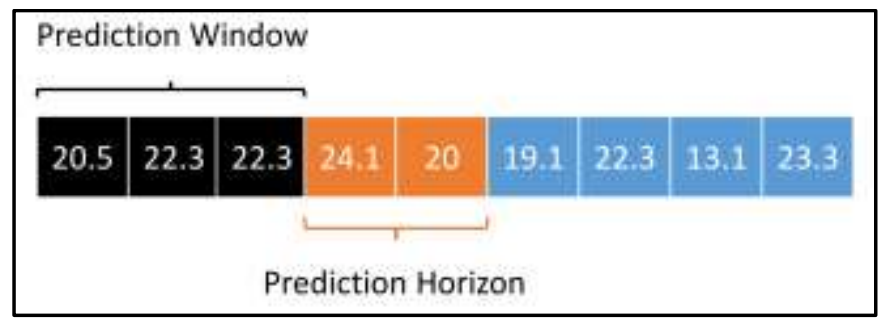

Source: Authors. 
In Figure 3, a prediction window of size $3(\mathrm{~J}=3)$ is used to generate an instance of forecasting. The prediction target of this instance is a horizon of size $2(\mathrm{H}=2)$. The prediction instances are generated in pairs \{Prediction window, horizon $\}$ by sliding the window in one by one steps until the end of the series is reached. Different sliding windows with $\mathrm{J}$ varying in a range and fixed $\mathrm{H}=24$ were implemented in order to find the best performance for each prediction model tested.

In order to compare the performances of both hyper-parameter and technique selection, for each tested configuration, 30 executions are performed. The values are displayed in both Tables 2 and 3 and represent the average RMSE and hypervolume, respectively.

\subsection{Recommendation}

The recommendation module was tested by taking the last forecast value for each appliance using their respective best prediction technique and parameters found. For each appliance, the respective forecast was used in the comfort objective function to find the best multi-objective optimization technique.

The multi-objective techniques tested for recommendation were Non-Sorted Genetic Algorithm II (Deb et al., 2002), Multi-objective Particle Swarm Optimization (Coello \& Lechuga,n.d.), Speed Constrained Multi-Objective Particle Swarm Optimization (Nebro et al., 2009), and Strength Pareto Evolutionary Algorithm 2 (Zitzler et al., 2001).

The final Pareto Front resulting from each execution was evaluated by the hypervolume (While et al., 2006). The metrics consist in calculating the area among all the solutions of the Pareto Front and a utopia point in the objective space. Figure 4 illustrates an example of the metrics.

Figure 4: Hypervolume (grey area). The $X$ and $Y$ axes are the values of the objective functions, $P$ are the non-dominated solutions from the resulting pareto front, and $r$ is a utopia point fixed for all the multi-objective techniques tested.

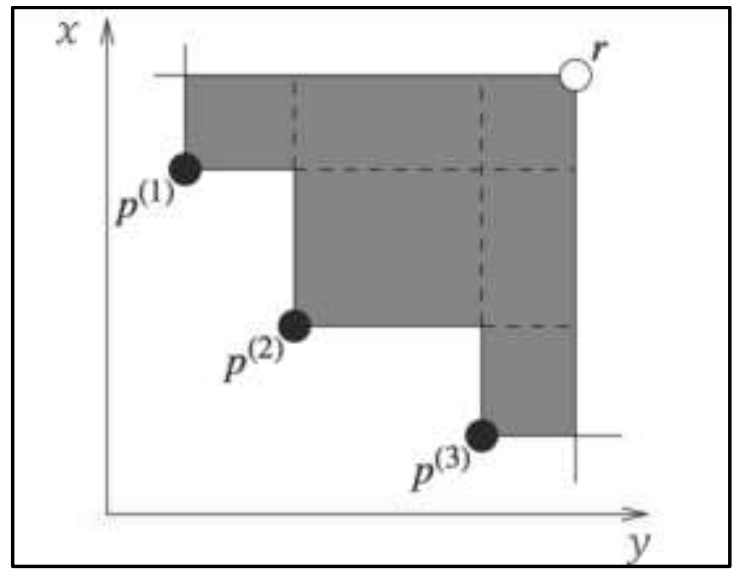

Source: pymoo.org

In Figure 4, the point $p$ represents solutions from the Pareto front generated by the multi-objective optimization technique, $r$ is a utopia point that is fixed for all the techniques being evaluated, and the calculated gray area is the hypervolume. This metric accounts for both spreading of the solutions and how optimal the solutions are in terms of minimizing the objective functions.

\section{Results and Discussion}

Data were available in a second -by -second sampling rate. However, forecasting one day ahead of usage on a persecond basis would result in a very high horizon and consequent uncertainty. In order to be able to generate forecasts for one 
day ahead, an hourly aggregation was performed. Despite the loss of information resulting from the aggregations, usage recommendations on a second by second basis might be too invasive and not easy to understand from the point of view of the user. The results of the experiments described above are described as follows.

\subsection{Forecasting}

Table 2 shows the average values of Root Mean Square Error values for each of the techniques tested (columns) and the forecasted appliances (rows).

Table 2: Mean RMSE (standard deviation, in parentheses) per technique for each appliance.

\begin{tabular}{|l|l|l|l|l|l|l|}
\hline Appliance & LSTM & ESN & ARIMA & GRU & SVR LIN & SVR POLY \\
\hline Fridge & $\mathbf{0 . 0 2 9}(\mathbf{0 . 0 0 2})$ & $0.033(0.010)$ & $0.351(0.081)$ & $0.032(0.003)$ & $0.036(0.002)$ & $0.084(0.023)$ \\
\hline Dryer & $0.008(0.007)$ & $\mathbf{0 . 0 0 6}(\mathbf{0 . 0 0 3})$ & $0.243(0.262)$ & $0.015(0.003)$ & $0.048(0.015)$ & $0.154(0.072)$ \\
\hline Coffee Machine & $\mathbf{0 . 0 7 4}(\mathbf{0 . 0 5 5})$ & $0.100(0.037)$ & $0.115(0.084)$ & $0.079(0.04)$ & $0.092(0.035)$ & $0.419(0.083)$ \\
\hline Kettle & $\mathbf{0 . 0 2 7}(\mathbf{0 . 0 2 3})$ & $0.031(0.017)$ & $0.089(0.056)$ & $0.039(0.028)$ & $0.032(0.019)$ & $0.095(0.021)$ \\
\hline Washing Machine & $\mathbf{0 . 0 2 2}(\mathbf{0 . 0 1 1})$ & $0.023(0.009)$ & $0.157(0.077)$ & $0.028(0.007)$ & $0.035(0.003)$ & $0.107(0.038)$ \\
\hline Desktop Computer & $\mathbf{0 . 0 1 5}(\mathbf{0 . 0 1 0})$ & $0.019(0.007)$ & $0.157(0.116)$ & $0.028(0.27)$ & $0.024(0.020)$ & $0.069(0.020)$ \\
\hline Freezer & $\mathbf{0 . 0 2 6}(\mathbf{0 . 0 0 1})$ & $0.032(0.009)$ & $0.103(0.081)$ & $0.029(0.005)$ & $0.028(0.003)$ & $0.084(0.022)$ \\
\hline
\end{tabular}

Source: Authors.

In the forecasting results, almost all tested techniques, except for ARIMA and SVR POLY, have accomplished similar performances.

As in (Kong et al., 2018), (Kumar et al., 2018), and (Kong et al., 2019), LSTM and GRU performed better, on average, for most of the appliances. Recurrent neural networks perform better when there is more uncertainty involved, than feed- forward neural networks, SVM and deterministic techniques like ARIMA, due to their capacity of dealing with the vanishing gradient problem in the training step. The vanishing gradient problem for forecasting does not apply for the proposed forecasting model in this work since the forecasting realized was a single step ahead.

In the experiments and conditions of this work, Echo State Networks, as in Shi et al. (2016), were also revealed as a reasonable choice for disaggregated residential load forecasting. 
Table 3: Nemenyi post hoc test pairwise $p$ values for the predictions of the appliance "Coffee Machine" with non-significant differences as NS.

\begin{tabular}{|c|c|c|c|c|c|}
\hline & ARIMA & ESN & GRU & LSTM & LIN SVR \\
\hline ESN & $\mathrm{p}<0.05$ & & & & \\
\hline GRU & $\mathrm{p}<0.01$ & NS & & & \\
\hline LSTM & $\mathrm{p}<0.01$ & NS & NS & & \\
\hline LIN SVR & $\mathrm{p}<0.01$ & NS & NS & NS & \\
\hline POLY SVR & NS & NS & NS & $\mathrm{p}<0.01$ & $\mathrm{p}<0.01$ \\
\hline
\end{tabular}

Source: Authors.

Despite the fact that some techniques have performed better than others, on average, most of them showed overlapping standard deviations when executed more than once. Therefore, the non-parametric Friedman test was performed with nemenyi post hoc in order to analyze if the pairs of techniques do or do not display meaningful differences between them. This test was performed only for the appliance "Coffee Machine", as it was the one that showed a larger RMSE average value for all techniques. In Table 3, comparing ARIMA with the other techniques, we can observe that, except for POLY SVR, the null hypothesis was rejected ( $\mathrm{p}$ value $<1 \times 10^{-6}$ ), which reveals statistical differences in the comparisons.

Regarding the other nemenyi post hoc comparisons in Table 3, except for ARIMA, the forecasting performed by ESN, GRU, LSTM, and Linear SVR are not statistically different from any other techniques. This behavior was similar for all the other appliances tested and, in the case of the appliance "Coffee Machine" in Table 3, there is a significant difference between LSTM and Polynomial SVR.

Since the horizon of prediction was fixed to 24 , in terms of window size, a range of values from one to twenty days was tested for each technique and each appliance. The best value was found by observing the smaller values of RMSE. For the appliance "Coffee Machine", the LSTM and ESN performed better with $J=336$ (two weeks of past usage data), GRU with $J=$ 288 (12 days of past usage data), and POLYNOMIAL and LINEAR SVR with $J=384$ (16 days of past usage data). In terms of the amount of past data needed to perform a forecast, deep learning was able to work with less data than the others, and GRU was able to generate its better forecasting with 12 days of past data. This behavior was similar for the other appliances, being different only in those cases in which the past data needed by GRU was the same as the LSTM and ESN.

\subsection{Recommendation}

The average hypervolume of the Pareto Fronts generated by the multi-objective techniques for recommendations are shown in Table 4. Those results show NSGA II as the best suited for generating recommendations, in the conditions of this work, for most of the appliances, except for "Coffee Machine" and "Kettle", where SPEA2 showed higher average hypervolume. 
Table 4: Average hypervolume (standard deviation, in parentheses) per technique for each appliance.

\begin{tabular}{|c|c|c|c|c|}
\hline Appliance & NSGA II & OMOPSO & SMPSO & SPEA2 \\
\hline Fridge & $\mathbf{0 . 6 4}(\mathbf{0 . 0 1})$ & $0.43(0.03)$ & $0.26(0.03)$ & $0.58(0.01)$ \\
\hline Dryer & $\mathbf{0 . 6 0}(\mathbf{0 . 0 1})$ & $0.45(0.02)$ & $0.32(0.02)$ & $0.56(0.01)$ \\
\hline Coffee Machine & $0.68(0.24)$ & $0.02(0.21)$ & $0.01(0.12)$ & $\mathbf{0 . 8 5}(\mathbf{0 . 2 1})$ \\
\hline Kettle & $0.50(0.17)$ & $0.12(0.06)$ & $0.006(0.03)$ & $\mathbf{0 . 7 6}(\mathbf{0 . 0 8})$ \\
\hline Washing Machine & $\mathbf{0 . 5 8}(\mathbf{0 . 0 0 8})$ & $0.51(0.01)$ & $0.44(0.01)$ & $0.54(0.01)$ \\
\hline Desktop Computer & $\mathbf{0 . 5 7}(\mathbf{0 . 0 0 9})$ & $0.49(0.01)$ & $0.43(0.01)$ & $0.53(0.01)$ \\
\hline Freezer & $\mathbf{0 . 6 0}(\mathbf{0 . 0 1})$ & $0.42(0.02)$ & $0.29(0.02)$ & $0.55(0.01)$ \\
\hline
\end{tabular}

Source: Authors.

As in the forecasting experiments, the Friedman test and nemenyi post hoc test were performed to find statistical differences between pairs of techniques, and the only non-significant differences from NSGA II and other techniques were for "Coffee Machine" (with results represented in Table 5) and 5 with SPEA2, meaning that for those two appliances, there was no difference in performance when using one or the other. For all the other appliances, there was a significant difference between NSGA II and all the other techniques tested, meaning that, in the conditions of the experiments realized in this work and regarding hypervolume, NSGA II is the best suited technique for generating recommendations.

Table 5: Nemenyi post hoc test for the recommendation hypervolumes from "Coffee Machine".

\begin{tabular}{|c|c|c|c|c|}
\hline & NSGA II & OMOPSO & SMPSO & SPEA2 \\
\hline OMOPSO & $\mathrm{p}<0.01$ & & & \\
\hline SMPSO & $\mathrm{p}<0.01$ & NS & & \\
\hline SPEA2 & NS & $\mathrm{p}<0.01$ & $\mathrm{p}<0.01$ & \\
\hline
\end{tabular}

Source: Authors.

In order to show the potential of energy saving of the recommendations generated for "Coffee Machine", the pareto front solutions of NSGA II, ordered by their energy consumption, were divided into three groups containing the same number of solutions. The average energy saving (compared with the forecasted value from the prediction module) of the three groups are shown in Table 6. The group in which the solutions are smaller in the power consumption objective (i.e. more similar to the forecasted usage) is denominated comfort group. The group with intermediate solutions is the medium group and the group with the solutions that are smaller in the objective of power usage is denominated economy group. 
Table 6: Potential energy saving of the recommendations from the Pareto Front generated for the "Coffee Machine".

\begin{tabular}{|l|c|}
\hline & \% of energy saving \\
\hline Comfort & 3.4 \\
\hline Medium & 7.9 \\
\hline Economy & 18.2 \\
\hline
\end{tabular}

Source: Authors.

In Table 6, the comfort group is the average energy savings of the solutions that save less energy and are more concerned with the comfort of the user; the medium group refers to the balance between user comfort and energy saving, and the economy group is the average energy saving of the recommendations that mostly affect the comfort of the user.

\section{Conclusion}

The present work recommends a system to generate more efficient ways for household appliance usage. This proposal took into consideration the dilemma between users' comfort and energy saving, becoming more realistic in terms of user experience and comfort interference. The choice of the technique for both forecasting and recommendation is problem dependent, as different techniques and models reveal the best options for different appliances. However, the choice of LSTM, ESN, or GRU, and a window of two weeks of past data as the only prediction for all the appliances would cover most of the scenarios with reasonable performance. In the same way, the choice of NSGA II for recommendations would perform well for all the appliances.

According to the experiments, deep learning based techniques have shown relatively better performance than some traditional regression techniques for forecasting the usage of appliances by the user in the near future. Also, for recommendations, the NSGA II has proven to be superior to the others in most cases.

The resultant recommendations from the Pareto Fronts can be proposed to Smart Home Users in different ways. For example, they can be transformed into a quota of appliance usage in the coming hours, or they can be part of an automatic system that turns off certain appliances automatically if the user has accepted the recommendation(s) to do so at a specific timestamp. Another way this recommendation could be followed is in the quantity of usage times of the appliance in case of a washing machine, for example.

The potential of energy saving was presented from one scenario (Washing Machine), in case of acceptance of its recommendations as having rates of $3.4 \%$ to $18.2 \%$ energy saving. In order to understand acceptability and for users to be able to follow recommendations, there is a need to test the operation of the proposed system(s) in a real home interaction with users.

As future works there is the need of investing in a hardware infrastructure to enable experiments approaching the acceptance of the recommendations by the users and perform a validation about the invasiveness to comfort of the users.

\section{Acknowledgement}

This research was partially supported by the Brazilian agency: Fundação de Amparo à Ciência e Tecnologia do Estado de Pernambuco (FACEPE).

\section{References}

Bergmeir, C., \& Benítez, J. M. (2012, May). On the use of cross validation for time series predictor evaluation. Information Sciences, 191, 192-213. 10.1016/j.ins.2011.12.028 
Box, G., Jenkins, G., \& Reinsel, G. (1994). Time series analysis: Forecasting and control. Prentice Hall. https://books.google.com.br/books?id=sRzvAAAAMAAJ

Cao, L. (2003, April). Support vector machines experts for time series forecasting. Neurocomputing, 51, 321-339. 10.1016/s0925-2312(02)00577-5

Cerqueira, V., Torgo, L., \& Mozetic, I. (2019). Evaluating time series forecasting models: An empirical study on performance estimation methods.

Chung, J., Gulcehre, C., Cho, K., \& Bengio, Y. (2014). Empirical evaluation of gated recurrent neural networks on sequence modeling.

Coello, C. C., \& Lechuga, M. (n.d.). MOPSO: a proposal for multiple objective particle swarm optimization. In Proceedings of the 2002 congress on evolutionary computation. CEC 02 (cat. no.02th8600). IEEE. 10.1109/cec.2002.1004388

Dao, V., Ishii, H., Takenobu, Y., Yoshizawa, S., \& Hayashi, Y. (2020, February). Intensive quadratic programming approach for home energy management systems with power utility requirements. International Journal of Electrical Power \& Energy Systems, 115, 105473. 10.1016/j.ijepes.2019.105473

Deb, K., Pratap, A., Agarwal, S., \& Meyarivan, T. (2002, April). A fast and elitist multiobjective genetic algorithm: NSGA II. IEEE Transactions on Evolutionary Computation, 6(2), 182-197. 10.1109/4235.996017

Din, G. M. U., Mauthe, A. U., \& Marnerides, A. K. (2018, March). Appliance Level short term load forecasting using deep neural networks. In 2018 international conference on computing, networking and communications (ICNC). IEEE. 10.1109/iccnc.2018.8390366

Dinh, H. T., Yun, J., Kim, D. M., Lee, K.-H., \& Kim, D. (2020). A home energy management system with renewable energy and energy storage utilizing main grid and electricity selling. IEEE Access, 8, 49436-49450. 10.1109/access.2020.2979189

Gajowniczek, K., \& Zabkowski, T. (2014). Short term electricity forecasting using individual smart meter data. Procedia Computer Science, 35, 589-597. 10.1016/j.procs.2014.08.140

Golmohamadi, H., Keypour, R., Bak-Jensen, B., \& Pillai, J. R. (2019, May). Optimization of household energy consumption towards day ahead retail electricity price in home energy management systems. Sustainable Cities and Society, 47, 101468. 10.1016/j.scs.2019.101468

Hochreiter, S., \& Schmidhuber, J. (1997). Long short term memory. Neural Computation, 9(8), 1735-1780. https://doi.org/10.1162/neco.1997.9.8.1735 10.1162/neco.1997.9.8.1735

Hossen, T., Nair, A. S., Noghanian, S., \& Ranganathan, P. (2018, September). Optimal operation of smart home appliances using deep learning. In 2018 north american power symposium (NAPS). IEEE. 10.1109/naps.2018.8600674

International Energy Agency, I. (2019). Energy.

Isnen, M., Kurniawan, S., \& Garcia-Palacios, E. (2020, February). A-SEM: An adaptive smart energy management testbed for shiftable loads optimisation in the smart home. Measurement, 152, 107285. 10.1016/j.measurement.2019.107285

Jaeger, H. (2001). The "echo state": Approach to analysing and training recurrent neural networks. GMD-Forschungszentrum Informationstechnik. https://books.google.com.br/books?id=-3NBHQAACAAJ

Khalid, A., Javaid, N., Guizani, M., Alhussein, M., Aurangzeb, K., \& Ilahi, M. (2018). Towards dynamic coordination among home appliances using multi-objective energy optimization for demand side management in smart buildings. IEEE Access, 6, 19509-19529. 10.1109/access.2018.2791546

Khalid, M. U., \& Javaid, N. (2018, April). An optimal scheduling of smart home appliances using heuristic techniques with real time coordination. In $20181 s t$ international conference on power, energy and smart grid (ICPESG). IEEE. 10.1109/ icpesg.2018.8384505

Khan, A. R., Mahmood, A., Safdar, A., Khan, Z. A., \& Khan, N. A. (2016, February). Load forecasting, dynamic pricing and DSM in smart grid: A review. Renewable and Sustainable Energy Reviews, 54, 1311-1322. 10.1016/j.rser.2015.10.117

Kleiminger, W., Beckel, C., \& Santini, S. (2015). Household occupancy monitoring using electricity meters. In Proceedings of the 2015 ACM international joint conference on pervasive and ubiquitous computing - UbiComp 15. ACM Press. 10.1145/2750858.2807538

Kong, W., Dong, Z. Y., Hill, D. J., Luo, F., \& Xu, Y. (2018, January). Short Term residential load forecasting based on resident behaviour learning. IEEE Transactions on Power Systems, 33(1), 1087-1088. 10.1109/tpwrs.2017.2688178

Kong, W., Dong, Z. Y., Jia, Y., Hill, D. J., Xu, Y., \& Zhang, Y. (2019, January). Short Term residential load forecasting based on LSTM recurrent neural network. IEEE Transactions on Smart Grid, 10(1), 841-851. 10.1109/tsg.2017.2753802

Kumar, S., Hussain, L., Banarjee, S., \& Reza, M. (2018, January). Energy load forecasting using deep learning approach-LSTM and GRU in spark cluster. In 2018 fifth international conference on emerging applications of information technology (EAIT). IEEE. 10.1109/eait.2018.8470406

Leitao, J., Gil, P., Ribeiro, B., \& Cardoso, A. (2020). A survey on home energy management. IEEE Access, 8, 5699-5722. 10.1109/access.2019.2963502

Liu, Y., Yuen, C., Yu, R., Zhang, Y., \& Xie, S. (2016, May). Queuing Based energy consumption management for heterogeneous residential demands in smart grid. IEEE Transactions on Smart Grid, 7(3), 1650-1659. 10.1109/tsg.2015.2432571

Merdanoğlu, H., Yakıcı, E., Doğan, O. T., Duran, S., \& Karatas, M. (2020, May). Finding optimal schedules in a home energy management system. Electric Power Systems Research, 182, 106229. 10.1016/j.epsr.2020.106229

Molla, T., Khan, B., Moges, B., Alhelou, H. H., Zamani, R., \& Siano, P. (2019). Integrated optimization of smart home appliances with cost effective energy management system. CSEE Journal of Power and Energy Systems, 5(2), 249-258. 
Monyei, C. G., Adewumi, A. O., Akinyele, D., Babatunde, O. M., Obolo, M. O., \& Onunwor, J. C. (2018, May). A biased load manager home energy management system for low cost residential building low income occupants. Energy, 150, 822-838. 10.1016/j.energy.2018.03.016

Nebro, A., Durillo, J., Garcia-Nieto, J., Coello, C. C., Luna, F., \& Alba, E. (2009, March). SMPSO: A new PSO-based metaheuristic for multi-objective optimization. In 2009 IEEE symposium on computational intelligence in multicriteria decision making. IEEE. 10.1109/mcdm.2009.4938830

Oprea, S.-V., Pîrjan, A., Căruțașu, G., Petroșanu, D.-M., Bâra, A., Stănică, J.-L., \& Coculescu, C. (2018, May). Developing a mixed neural network approach to forecast the residential electricity consumption based on sensor recorded data. Sensors, 18(5), 1443. 10.3390/s18051443

Shafie-Khah, M., \& Siano, P. (2018, February). A stochastic home energy management system considering satisfaction cost and response fatigue. IEEE Transactions on Industrial Informatics, 14(2), 629-638. 10.1109/tii.2017.2728803

Shakeri, M., Shayestegan, M., Reza, S. S., Yahya, I., Bais, B., Akhtaruzzaman, M., ... Amin, N. (2018, September). Implementation of a novel home energy management system (HEMS) architecture with solar photovoltaic system as supplementary source. Renewable Energy, 125, 108-120. 10.1016/j.renene.2018.01.114

Shareef, H., Ahmed, M. S., Mohamed, A., \& Hassan, E. A. (2018). Review on home energy management system considering demand responses, smart technologies, and intelligent controllers. IEEE Access, 6, 24498-24509. 10.1109/access.2018.2831917

Shi, G., Liu, D., \& Wei, Q. (2016, December). Energy consumption prediction of office buildings based on echo state networks. Neurocomputing, 216, 478488. 10.1016/j.neucom.2016.08.004

Veras, J., Silva, I., Pinheiro, P., Rabêlo, R., Veloso, A., Borges, F., \& Rodrigues, J. (2018, September). A multi-objective demand response optimization model for scheduling loads in a home energy management system. Sensors, 18(10), 3207. 10.3390/s18103207

While, L., Hingston, P., Barone, L., \& Huband, S. (2006, February). A faster algorithm for calculating hypervolume. IEEE Transactions on Evolutionary Computation, 10(1), 29-38. https://doi.org/10.1109/tevc.2005.851275 10.1109/tevc.2005.851275

Yuce, B., Rezgui, Y., \& Mourshed, M. (2016, January). ANN-GA smart appliance scheduling for optimised energy management in the domestic sector. Energy and Buildings, 111, 311-325. 10.1016/j.enbuild.2015.11.017

Zitzler, E., Laumanns, M., \& Thiele, L. (2001). Spea2: Improving the strength pareto evolutionary algorithm. 10.3929/ETHZ-A-004284029 